\title{
Class I DISARM provides anti-phage and anti-conjugation activity by unmethylated DNA recognition
}

Cristian Aparicio-Maldonado ${ }^{1,2}$, Gal Ofir $^{3}$, Andrea Salini $^{1}$, Rotem Sorek ${ }^{3}$, Franklin L. Nobrega $^{4, *}$, Stan J.J. Brouns ${ }^{1,2, *}$

${ }^{1}$ Department of Bionanoscience, Delft University of Technology, Delft, 2629HZ, Netherlands

${ }^{2}$ Kavli Institute of Nanoscience, Delft University of Technology, Delft, 2629HZ, Netherlands

${ }^{3}$ Department of Molecular Genetics, Weizmann Institute of Science, Rehovot 7610001, Israel

${ }^{4}$ School of Biological Sciences, University of Southampton, Southampton, SO17 1BJ, United Kingdom

* To whom correspondence should be addressed. Tel: +31 15278 3920; Email: stanbrouns@gmail.com

* Correspondence may also be addressed to: Tel: +44 238059 7651; Email: F.Nobrega@ @oton.ac.uk

Present Address: Cristian Aparicio-Maldonado, School of Biological Sciences, University of Southampton, Southampton, SO17 1BJ, United Kingdom

Present Address: Gal Ofir, Department of Molecular Biology, Max Planck Institute for Developmental Biology, Tübingen, 72076, Germany. 


\section{ABSTRACT}

Bacteriophages impose a strong evolutionary pressure on microbes for the development of

3 mechanisms of survival. Multiple new mechanisms of innate defense have been described recently,

4 with the molecular mechanism of most of them remaining uncharacterized. Here, we show that a

5 Class 1 DISARM (defense island system associated with restriction-modification) system from

$6 \quad$ Serratia sp. provides broad protection from double-stranded DNA phages, and drives a population

7 of single-stranded phages to extinction. We identify that protection is not abolished by deletion of

8 individual DISARM genes and that the absence of methylase genes $d r m M I$ and $d r m M I I$ does not

9 result in autoimmunity. In addition to antiphage activity we also observe that DISARM limits

10 conjugation, and this activity is linked to the number of methylase cognate sites in the plasmid.

11 Overall, we show that Class 1 DISARM provides robust anti-phage and anti-plasmid protection

12 mediated primarily by $\operatorname{drm} A$ and $\operatorname{drm} B$, which provide resistance to invading nucleic acids using a

13 mechanism enhanced by the recognition of unmethylated cognate sites of the two methylases

$14 d r m M I$ and drmMII.

15 Keywords: DISARM, DNA methylation, bacteriophage, bacterial defense system, conjugation. 


\section{INTRODUCTION}

The arms race between prokaryotes and bacteriophages drives their co-evolutionary dynamics and has led to the evolution of multiple antiviral defense mechanisms in prokaryotes that are

19 collectively known as the prokaryotic immune system (1). Most prokaryotic defenses are innate,

20 acting via recognition of general signals that are not shared by the microbial cell. A well-known

21 example of innate defense are the highly abundant restriction-modification (R-M) systems (2) that

22 cleave phage nucleic acids at sequence motifs protected in the host chromosome by epigenetic

23 modifications (3). Recently, the analysis of genomic neighborhoods of known defense systems

24 revealed multiple new anti-phage systems (4-12), among which the defense island system

25 associated with restriction-modification (DISARM) (13).

26 The DISARM system is composed of three-core genes: gene $\operatorname{drmA}$ with a helicase domain

27 (pfam00271); gene $d r m B$ with a DUF1998 domain (pfam09369, helicase-associated); and gene

$28 d r m C$ with a phospholipase D (PLD) domain (pfam13091). In the uncharacterized Class 1

29 DISARM systems, this core triplet is preceded by the SNF2-like helicase drmD (pfam00176), and

30 the DNA adenine N6 methylase $d r m M I$ (pfam13659) (13). Class 2 DISARM systems contain, in

31 addition to the core gene triplet, the DNA 5-cytosine methylase drmMII (pfam00145) and, on

32 occasion, the gene of unknown function $d r m E$. The Class 2 DISARM system was shown to use

33 methylation of specific host motifs to mark self-DNA, akin to R-M systems, but its molecular

34 mechanism remains largely unknown. Unlike R-M systems, DISARM seems to not fully depend

35 on the sequence motif identified by the methylase to interfere with the incoming DNA; importantly,

36 the candidate nuclease of the system $(\operatorname{drm} C)$ was found dispensable for resistance and its activity

37 is yet unknown. 
38 In some cases, Class 1 DISARM systems are accompanied by an additional cytosine methylase

39 drmMII gene (13). Here we characterized such a Class 1 DISARM system present in Serratia sp.

40 SCBI (Figure 1A). We found that Class 1 DISARM provides broad anti-phage and anti-

41 conjugation activity independent of methylation status of the incoming DNA, and drives a

42 population of chronic infecting phages to extinction. Unlike Class 2 DISARM and R-M systems,

43 the methylases of Class 1 DISARM only partially methylate adenine and cytosine bases of the host

44 DNA at motifs ACACÁG and MTCGAK, and the absence of the methylases does not result in

45 autoimmunity. Overall, our results show that DISARM combines methylation and non-methylation

46 signals to provide protection against invader DNA, establishing a clear distinction from R-M

47 systems. 


\section{MATERIAL AND METHODS}

\section{Bacterial strains and growth conditions}

50 Escherichia coli strains DH5a, BL21-AI, JM109, ER2738, WG5, C3000, and S17-1 were

51 cultured in Lysogeny Broth (LB) media at $37^{\circ} \mathrm{C}$. For solid media experiments, LB was

52 supplemented with $1.5 \%(\mathrm{w} / \mathrm{v})$ agar (LBA) and the cultures incubated overnight at $37^{\circ} \mathrm{C}$. When

53 required, media was supplemented with antibiotics at the following final concentrations: 100

$54 \mu \mathrm{g} / \mathrm{mL}$ ampicillin, $50 \mu \mathrm{g} / \mathrm{mL}$ kanamycin, $50 \mu \mathrm{g} / \mathrm{mL}$ streptomycin, $50 \mu \mathrm{g} / \mathrm{mL}$ gentamicin, and 25

$55 \mu \mathrm{g} / \mathrm{mL}$ chloramphenicol. Gene expression was induced with $1 \mathrm{mM}$ Isopropyl $\beta$-d-1-

56 thiogalactopyranoside (IPTG) and 0.2\% (w/v) L-arabinose. The Serratia sp. SCBI strain (South

57 African Caenorhabditis briggsae Isolate) was grown aerobically in LB media at $30^{\circ} \mathrm{C}$ with shaking

58 at $180 \mathrm{rpm}$.

59 Phage cultivation

60 E. coli phages were propagated using their host strain (Supplementary Table S1) as described

61 previously (14). Briefly, bacterial cultures at early exponential growth phase (0.3-0.4 OD 600$)$ were

62 infected with a phage lysate and incubated overnight at $37^{\circ} \mathrm{C}$ with shaking. Cultures were spun

63 down and the supernatant filtered $(0.2 \mu \mathrm{m}$ PES $)$ and stored at $4{ }^{\circ} \mathrm{C}$ until use. When required, phages

64 were concentrated by adding PEG-8000 and $\mathrm{NaCl}$ at final concentrations of $100 \mathrm{mg} / \mathrm{mL}$ and $1 \mathrm{M}$,

65 respectively. The suspension was incubated overnight at $4^{\circ} \mathrm{C}$, centrifuged at $11,000 \times g$ at $4^{\circ} \mathrm{C}$ for

$66 \mathrm{~h}$, and the phage-containing pellet was re-suspended in $\mathrm{SM}$ buffer $(100 \mathrm{mM} \mathrm{NaCl}, 8 \mathrm{mM}$

$67 \mathrm{MgSO}_{4} .7 \mathrm{H}_{2} \mathrm{O}$, and $50 \mathrm{mM}$ Tris- $\mathrm{HCl} \mathrm{pH} \mathrm{7.5).} \mathrm{The} \mathrm{phage} \mathrm{titer} \mathrm{was} \mathrm{determined} \mathrm{using} \mathrm{the} \mathrm{small} \mathrm{drop}$

68 plaque assay method as described by Mazzocco and colleagues (15). E. coli strain BL21-AI was

69 used for titering and performing assays of phages T1, T3, T4, T7, Lambda-vir, Myo21S, and 
70 Myo22L, strain WG5 was used for MS2, and strain C3000 was used for PhiX174. Phage M13 was

71 titered using E. coli strain ER2738 and 0.6\% LBA supplemented with $1 \mathrm{mM}$ IPTG and $200 \mu \mathrm{g} / \mathrm{mL}$

72 X-gal. Assays of M13 phage were performed in strain JM109 (DE3).

\section{Cloning of Class 1 DISARM and mutants}

74 Plasmids and primers used in this study are listed in Supplementary Table S2 and

75 Supplementary Table S3, respectively. The complete Class 1 DISARM system and different

76 combinations of its genes were amplified by PCR from Serratia sp. SCBI genomic DNA with

77 primers indicated in Supplementary Table S3 and using Q5 DNA Polymerase (New England

78 Biolabs) according to the manufacturer's instructions. The PCR products were cloned into the

79 plasmid backbones using the NEBuilder HiFi DNA Assembly Cloning Kit (New England Biolabs)

80 following manufacturer's instructions. Plasmid pDIS_3 was used as a template for PCR with

81 primers listed in Supplementary Table S3 to construct pDIS_5, pDIS_6 and pDIS_7 by restriction

82 cloning. All plasmids were confirmed by Sanger sequencing (Macrogen) and transformed into

83 strains E. coli BL21-AI and E. coli JM109(DE3) for the assays described below.

\section{Phage infection growth curves}

85 Bacterial cultures with the DISARM system or empty vector were grown to early exponential 86 phase (OD 600 of $0.2-0.3$ ), induced with IPTG and L-arabinose, and grown for 90 min at $37{ }^{\circ} \mathrm{C}$ and

$87180 \mathrm{rpm}$. The cultures were normalized to an $\mathrm{OD}_{600}$ of 0.5 (approximately $1 \times 10^{8} \mathrm{CFU} / \mathrm{ml}$ ) and 190

$88 \mu \mathrm{l}$ were dispensed into wells of 96-well microtiter plates. Then, $10 \mu \mathrm{l}$ of phage suspension were

89 added to the wells at different multiplicity of infection (MOI), and bacterial growth was followed

90 in an $\mathrm{EPOCH} 2$ microplate reader with $\mathrm{OD}_{600}$ measurements every $10 \mathrm{~min}$ at $37^{\circ} \mathrm{C}$ with constant

91 shaking. 


\section{Phage replication over time}

93 Bacterial cultures were prepared as above and infected with phages at different MOIs. Cultures

94 were incubated at $37^{\circ} \mathrm{C}$ with shaking at $180 \mathrm{rpm}$, and phage titers were measured over time using

95 the small drop plaque assay method using the wild type strain as the host.

\section{Methylation-sensitive DNA sequencing}

97 Genomic DNA was extracted from the wild-type strain and strains containing the complete

98 DISARM system or its methylases, using the phenol chloroform method as described before (16)

99 with some modifications (17). Briefly, cultures at exponential phase were induced and incubated

100 overnight. Bacterial cells were pelleted, re-suspended in TE buffer, and treated with RNase I and

101 lysozyme at $1 \mu \mathrm{g} / \mathrm{ml}$ for $1 \mathrm{~h}$, followed by proteinase $\mathrm{K}$ at $50 \mu \mathrm{g} / \mathrm{ml}$ for $1 \mathrm{~h}$. DNA was extracted twice

102 with phenol:chloroform (1:1) and precipitated by adding $300 \mathrm{mM}$ sodium acetate $\mathrm{pH} 5.2$ and two

103 volumes of absolute ethanol. After incubation at $-20^{\circ} \mathrm{C}$ for $1 \mathrm{~h}$ or overnight, the DNA was pelleted

104 by centrifugation at $21,000 \times g$, and the pellet re-suspended in nuclease-free water. The DNA was

105 quantified using Qubit dsDNA HS Assay kit (Invitrogen) and the quality assessed using Nanodrop

106 (Thermo Scientific).

108 (SMRT) sequencing technology to detect DNA methylation sites (18). DNA libraries were

109 prepared using the SMRTbell® Express Template Prep Kit 2.0 and the Barcoded Overhang

110 Adapter kit according to the manufacturer instructions. Sequencing was performed on a PacBio

111 Sequel platform using Sequel sequencing kit 3.0. The data were analyzed using the Base

112 Modification and Motif Analysis module of the SMRT-Link v7.0.1 software to detect methylation

113 motifs. 
114 Bisulfite library preparation and sequencing was done as previously described (13). Sequencing

115 results were analyzed using Bismark v0.20.1 (Krueger \& Andrews 2011

116 https://doi.org/10.1093/bioinformatics/btr167) to identify methylated cytosines. The sequence

117 neighborhood of methylated cytosines was analyzed using Weblogo (Crooks et al. 2004) to

118 determine the methylation motif.

119 Raw sequencing data were deposited in Figshare for PacBio

120 (doi.org/10.6084/m9.figshare.17295215) and Bisulfite (doi.org/10.6084/m9.figshare.17295212)

121 sequencing.

\section{Construction of motif-containing conjugative plasmids}

123 Synthetic constructs (Integrated DNA Technologies) of tetracycline-regulated YFP and a motif-

124 adaptable module (MAM) (Supplementary Table S4) were introduced into pSEVA_331 by

125 restriction cloning. Putative DISARM motifs were removed from the backbone sequence of

126 pSEVA_331 by replacing these regions with synthetic constructs (Supplementary Table S4). For

127 this, pSEVA_331 and the synthetic constructs were amplified using primers in Supplementary

128 Table S3 and Q5 DNA Polymerase (New England Biolabs), and cloned by restriction digest using

129 the enzymes indicated in Supplementary Tables S3 and S4, giving rise to plasmid pCONJ.

130 Plasmids pCONJ_1 to pCONJ_8 (Supplementary Table S2) were created by cloning synthetic

131 constructs (Supplementary Table S4) containing different motif combinations into the MAM

132 regions of pCONJ using restriction digest as above. All plasmid constructs were confirmed by

133 sequencing (Macrogen) and transformed into E. coli BL21-AI. 


\section{Conjugation efficiency}

135 The E. coli strain S17-1 containing variants of the plasmid pCONJ (Supplementary Table S2)

136 was used as the donor strain. Cells were grown to exponential phase and induced when necessary.

137 Approximately $5 \times 10^{8}$ cells of both donor strain and the DISARM or wild-type strain (recipient)

138 were spun down and re-suspended in $5 \mathrm{ml}$ of fresh LB media. The strains were combined in equal

139 counts in a final concentration of $1 \times 10^{8} \mathrm{CFU} / \mathrm{mL}$. After gently mixing, cells were pelleted at 2,000

$140 \times g$ for $10 \mathrm{~min}$ at room temperature, and incubated at $37^{\circ} \mathrm{C}$ for $4 \mathrm{~h}$ without shaking. The cell mixture

141 was plated onto LBA plates containing different antibiotics to determine the proportion of recipient

142 cells that acquired the plasmid from the donor strain. Conjugation efficiency was estimated as the

143 ratio of plasmid-acquisition events versus the total number of recipient strain cells.

\section{Statistical analysis}

145 The average values of three biological replicates were reported in the result and supplementary

146 sections. Unpaired two-tailed t Test and one-way analysis of variance (ANOVA) with Dunnett's

147 post-hoc multiple comparison test were used to compare the means between groups. Confidence

148 intervals were set at $95 \%(*=\mathrm{p}<0.05 ; * *=\mathrm{p}<0.001 ; * * *=\mathrm{p}<0.0001)$. Statistical analysis was

149 performed using GraphPad Prism version 5.0 for Windows. 


\section{RESULTS}

\section{Class 1 DISARM protects against widely diverse DNA phages}

152 To determine whether the predicted Class 1 DISARM system from Serratia sp. SCBI provides

153 protection from phage infection, we transplanted the six genes of the system into E. coli BL21-AI

154 (Figure 1A). We then challenged the DISARM-containing strain (DISARM (+)) with

155 Caudovirales of three morphologies (sipho-, myo-, and podophages) (19) at different MOI. Class

1561 DISARM shows clear anti-phage protection against all phages tested, by preventing or delaying

157 the collapse of the bacterial population upon phage infection even at high MOI (Figure 1B,

158 Supplementary Figure S1). To quantify the level of protection, we measured the efficiency of

159 plating (EOP) of the same set of phages on the DISARM (+) strain in comparison to control

160 (DISARM (-)) cells. Class 1 DISARM provided significant protection against phages T1, T4, T7,

161 Nami and phiX174 (Figure 1C).

162 Overall, both liquid and solid media assays demonstrate the broad anti-phage activity provided 163 by Class 1 DISARM.

\section{Class 1 DISARM can drive a phage population with chronic lifestyle to extinction}

165 We next investigated the effects of the Class 1 DISARM on the propagation of a phage and 166 accumulation of active phage in the cell culture. For this, we measured the phage titers periodically 167 upon infection of DISARM (+) or DISARM (-) strains with T1 or Nami phages. The number of 168 infectious T1 phages in the population is reduced by approximately 3 orders of magnitude in 169 DISARM containing strains from $30 \mathrm{~min}$ on, reaching a maximum reduction of $5.6 \times 10^{4}$ fold at 180 
170 minutes post-infection (Figure 1D). DISARM also inhibits the propagation of phage Nami, with a

171 maximum reduction of approximately 50-fold at 180 minutes post-infection (Figure 1D).

172 Virulent Caudovirales follow a lytic life cycle where the production of phages occurs typically

173 within 10-30 minutes after the ejection of the phage genome, ultimately resulting in cell death for

174 the release of the newly formed phage particles. Phages that follow a chronic life cycle are able to

175 produce new phages continuously without causing cell death, with the new virions extruding out

176 of the cell (20). To investigate the effect of Class 1 DISARM on the propagation of a phage

177 population with chronic lifestyle, we monitored the phage titers of a culture infected with the

178 single-stranded DNA (ssDNA) inovirus M13. We observed a rapid decrease of the number of 179 phages, with DISARM containing strains producing no more phage after 24h (Figure 1D), which 180 was not observed for any of the Caudovirales tested. To understand if the strong activity of Class 181 DISARM against phage M13 is a consequence of its chronic lifestyle or the type of genetic 182 material (ssDNA versus the double-stranded DNA of Caudovirales), we additionally tested

183 DISARM against ssDNA phage phiX174. PhiX174 uses a mechanism of phage DNA replication

184 similar to that of phage M13 $(21,22)$, but follows a lytic life cycle. The protective effect of 185 DISARM against infection by phiX174 was similar to that obtained for the dsDNA phages 186 (Supplementary Figure S3A), suggesting that the strong effect of DISARM against phage M13 187 is not related to the single stranded nature of the phage genome in the phage particle. We further 188 tested the protective effect of Class 1 DISARM against single-stranded RNA (ssRNA) phage MS2 189 but observed no protection (Supplementary Figure S3B), suggesting that Class 1 DISARM is a 190 DNA-directed defense system. In summary, Class 1 DISARM reduces the number of infectious 191 phages produced over time in a bacterial culture, and is able to completely abolish the propagation 192 of chronic phage M13. 


\section{Class 1 DISARM provides protection independent of methylation status}

194 We next studied the essentiality of the individual genes of the Class 1 DISARM system in anti-

195 phage protection. First, we used EOP assays to challenge cells harboring the complete or partial

196 DISARM system. We observed that DISARM provides protection against infection by dsDNA

197 phages T1 and Nami, and that some of the DISARM genes can be deleted while retaining either

198 full or partial protection (Figure 2A, Supplementary Figure S4A). To better evaluate gene

199 essentiality in Class 1 DISARM, we followed phage propagation over time in liquid culture of cells

200 harboring the complete or partial DISARM system. Results are shown in Figure 2B,C and

201 Supplementary Figure S4B as the increase of phage titer over time at the time point where

202 DISARM achieved the strongest effect compared to the titer at the start of infection. In contrast to

203 what was previously observed for Class 2 DISARM systems (13), deletion of core genes $d r m A B C$

204 did not abolish the full protective effect for both phages T1 and M13, and only the additional

205 deletion of $d r m M I I$ resulted in complete loss of protection with full restoration of the phage

206 replication capacity. For phage Nami, deletion of core $d r m A B C$ or deletion of $d r m D$ and $d r m M I$

207 resulted in almost complete loss of protection (Figure 2B), with full restoration of phage

208 replication being achieved with their combined deletion. Importantly, we observed that $d r m C$ is

209 not required for the anti-phage activity (Supplementary Figure S5) of Class 1 DISARM, as

210 previously observed for Class 2 DISARM (13).

211 Overall, we observed that Class 1 DISARM phage protection is relatively robust to deletion of

212 individual genes of the operon. 
215 sequenced the genomes of the wild type strain, the DISARM (+) strain, and the strain containing

216 one or both methylases using sequencing methods sensitive to epigenetic marks. The adenine

217 methylation by drmMI was characterized by PacBio sequencing and revealed an N6-

218 methyladenosine (m6A) modification of the ACACAGG motif (methylated base underlined, Figure

219 3A, Supplementary Table S5). The cytosine methylation by drmMII was characterized by

220 bisulfite sequencing and revealed a 5-methylcytosine (5mC) modification of MTCGAK motifs

221 (methylated cytosine underlined, Figure 3B, Supplementary Table S5), which is a distinct motif

222 from the $5 \mathrm{mC}$ modification in CWGG motifs reported for the Class 2 DISARM system of

223 Bacillus subtilis (13). The completeness of modification by the methylases was higher in the

224 presence of the full DISARM system $(84.3 \% \mathrm{~m} 6 \mathrm{~A}$, and $67.0 \% 5 \mathrm{mC})$ than in the presence of the

225 two methylases alone (56.5\% m6A, and 34.0\% 5mC), suggesting some form of synergetic effect

226 by the methylase pair (Figure 3D). Curiously, the presence of both $d r m M I$ and $d r m M I I$ increased

227 the m6A methylation ratio of $d r m M I$ to $73.9 \%$, but had no effect on $5 \mathrm{mC}$ methylation by $d r m M I I$.

228 No relation was observed between the distance of methylated or unmethylated ACACAG and

229 MTCGAK motifs, suggesting that their methylation status was independent of their relative

230 location in the genome. In summary, the Class 1 DISARM system of Serratia sp. SCBI uses both

231 adenine and cytosine methylation to modify specific motifs in the bacterial chromosome.

232 Class 1 DISARM displays anti-conjugation activity dependent on number of cognate sites

233 To determine the influence of the methylation pattern of the invading DNA on the level of 234 protection by Class 1 DISARM, we performed conjugation assays with plasmid pCONJ which 
235 does not contain ACACÁG or MTCEGAK motifs (Supplementary Table S2). We first assessed

236 the protection provided by DISARM towards conjugation of the unmethylated pCONJ, and

237 observed an approximately 47-fold reduction in conjugation efficiency in the presence of DISARM

238 (Supplementary Figure S6A). Next, we engineered different versions of pCONJ with variable

239 numbers of DISARM methylation motifs and performed conjugation assays in the DISARM (+)

240 and DISARM (-) strains as recipients. The conjugation efficiency of non-methylated pCONJ in the

241 DISARM (+) strain decreased moderately with increasing numbers of ACACAG and TCGA motifs

242 present in the plasmid, up to 12-fold (Figure 3E, Supplementary Figure S6B). The protective

243 effect against plasmid conjugation is only slightly stronger when both types of motifs are present.

244 Next, we compared the conjugation efficiency of pCONJ originated from cells expressing

245 DISARM methylases DrmMI and DrmMII to that of pCONJ containing non-methylated motifs

246 (Figure 3F). The conjugation efficiency of pCONJ was drastically elevated by 134- and 82-fold

247 when the donor strain contained both DrmMI and DrmMII, supporting the role of methylation as

248 an off switch for DISARM activity. The control plasmid, in which no ACACAG and MTCGAK

249 motifs were added, displayed a 21-fold increase in conjugation efficiency with both methylases

250 present in donor strain possibly due to effects of induction of gene expression of both methylases

251 in the donor. Overall, the conjugation assays demonstrate that Class 1 DISARM displays anti-

252 conjugation activity that is enhanced by the presence of unmethylated forms of both methylase

253 motifs in the plasmid. 


\section{DISCUSSION}

Here, we show that Class 1 DISARM of Serratia sp. SCBI provides broad protection against phages and plasmid conjugation using a mechanism of incoming nucleic acid detection which is

257 enhanced by the recognition of unmethylated cognate sites of the two methylases $d r m M I$ and $258 d r m M I I$. In Class 2 DISARM systems, the deletion of genes $\operatorname{drmA}$, $d r m B$, or $\operatorname{drmE}$ resulted in 259 complete loss of protection (13). The Class 1 DISARM system of Serratia sp. provides a more

260 robust protection, in which the deletion of individual genes is not sufficient to abolish the protective

261 effect of the system against some phages (e.g. T1 and M13). We observed that the number of 262 unmethylated motifs present in the incoming foreign DNA increased the protective effect of 263 DISARM against incoming invaders.

We found that the protection against phages was not scaled to the number of $d r m M I$ and $d r m M I I$

265 sites in their genomes (Supplementary Table S6), suggesting also other factors at play. Similarly,

266 the number of methylation sites on phage genomes did not affect the protection for the BREX (23)

267 system, and the same was suggested for Class 2 DISARM (13). This is markedly distinct from

268 tested R-M systems in which restriction (and therefore protection) is dependent on the number of

269 methylation sites in the invader's DNA (24). This suggests that DISARM and BREX use

270 mechanisms to identify invader DNA distinct from those of R-M systems, and which may prevent

271 strong negative selection for specific methylation motifs. It is also possible that the intrinsic

272 methylation patterns of phage DNA affect defense by DISARM, as observed previously for R-M

273 and BREX systems $(24,25)$.

274 The use of a molecular mechanism distinct from classical R-M systems is further supported by 275 the lack of autoimmunity in cells in the absence of drmMI and $d r m M I I$ (Supplementary Figure 
276 S7), contrary to R-M systems where this results in cleavage of the bacterial DNA (26). This is also

277 consistent with the fact that not all motifs in the bacterial genome of the transplanted host are

278 methylated by the DISARM methylases, unlike the almost complete motif methylation observed

279 in R-M. This suggests a tight regulation of the defense activity of Class 1 DISARM that seems to

280 result from the physical occlusion of the DNA entry site of the DrmAB complex by the trigger

281 loop, which is removed upon presence of a 5' ssDNA end (27). Interestingly, the methylase drmMII

282 of Class 2 DISARM provides almost complete methylation of motifs in the bacterial chromosome

283 and its absence was found to be deleterious to the cells (13). Differences in the molecular

284 mechanisms of Class 1 and Class 2 DISARM likely result from the use of distinct methylases. We

285 found that Serratia's Class 1 DISARM system has the unique feature of combining methylation of

286 both palindromic and non-palindromic motifs in the bacterial chromosome. Akin to BREX and R-

287 M type I and III systems, DrmMI of Class 1 DISARM methylates a non-palindromic site

288 (ACACAG). The modification occurs at the adenine in the fifth position of the recognition site, as

289 previously reported for the BREX site TAGGAG (8). Because the recognition motif of DrmMI is

290 non-palindromic, only one DNA strand will be methylated. Some R-M systems (e.g. type III and

291 type ISP) maintain the epigenetic marks by requiring interactions between different sites (28), but

292 it is unclear if DISARM and BREX use similar mechanisms.

Contrary to DrmMI, the DrmMII methylase of Class 1 DISARM modifies a degenerate

294 palindromic site (MTCGAK) in the bacterial chromosome. The methylation site of DrmMII of the

295 Bacillus paralicheniformis Class 2 DISARM system was also shown to be palindromic, although

296 of an unrelated sequence (CNGG) (13), much like the methylases of R-M type II systems.

297 Interestingly, DrmMII (palindromic motif) alone was shown to provide anti-phage activity against

298 the chronic infecting phage M13 and phage T1, possibly by interfering with the phage replication 
299 cycle as observed previously (29). DrmMI (non-palindromic motif) had no observable impact on

300 anti-M13 activity, as previously observed also for the BREX system (non-palindromic motif).

301 DrmABC without any of the methylases also provides protection against M13. It is possible that

302 the strong activity of DISARM against phage M13 results from the added effect of DrmABC and

303 a possible direct effect of DrmMII methylation of palindromic sites on DNA replication of M13.

304 In conclusion, we show that Class 1 DISARM systems are effective on viral and plasmid DNA,

305 and show enhanced protection against invader DNA when unmethylated cognate DNA motifs are

306 present (Figure 4). The mechanism of DISARM is remarkably robust with many of its components

307 playing an enhancing but non-essential role. 


\section{ACKNOWLEDGEMENT}

309 The authors thank Zohar Mukamel and the Weizmann Life Science Core Facilities for help with

310 bisulfite and PacBio sequencing, respectively.

\section{FUNDING}

312 This work was supported by the Netherlands Organisation for Scientific Research (NWO) with

313 Vici grant VI.C182.027 to S.J.J.B and Veni grant 016.Veni.181.092 to F.L.N.

\section{AUTHOR CONTRIBUTIONS}

315 S.J.J.B. and F.L.N. conceived the research. C.A.M. and A.S. performed the experiments. G.O. and

316 R.S. performed the methylation-sensitive sequencing and corresponding data analysis. All authors

317 contributed to data analysis and discussed the results. C.A.M. wrote the manuscript. F.L.N. and

318 S.J.J.B. reviewed and edited the manuscript with input from all authors. All authors approved the

319 manuscript. 


\section{REFERENCES}

321 1. Labrie,S.J., Samson,J.E. and Moineau,S. (2010) Bacteriophage resistance mechanisms. Nat. Rev.

$322 \quad$ Microbiol., 8, 317-327.

323 2. Tesson,F., Hervé,A., Touchon,M., Humières,C. and Cury,J. (2021) Systematic and quantitative

324 view of the antiviral arsenal of prokaryotes. bioRxiv, 10.1101/2021.09.02.458658.

3. Tock,M.R. and Dryden,D.T.F. (2005) The biology of restriction and anti-restriction. Curr. Opin. Microbiol., 8, 466-472.

4. Doron,S., Melamed,S., Ofir,G., Leavitt,A., Lopatina,A., Keren,M., Amitai,G. and Sorek,R. (2018) Systematic discovery of antiphage defense systems in the microbial pangenome. Science, 4120, 0-12.

5. Gao,L., Altae-Tran,H., Böhning,F., Makarova,K.S., Segel,M., Schmid-Burgk,J.L., Koob,J., Wolf,Y.I., Koonin,E. V. and Zhang,F. (2020) Diverse enzymatic activities mediate antiviral immunity in prokaryotes. Science, 1084, 1077-1084.

6. Kronheim,S., Daniel-Ivad,M., Duan,Z., Hwang,S., Wong,A.I., Mantel,I., Nodwell,J.R. and Maxwell,K.L. (2018) A chemical defence against phage infection. Nature, 564, 283-286. Argonaute. Nature, 507, 258-261.

8. Goldfarb,T., Sberro,H., Weinstock,E., Cohen,O., Doron,S., Charpak-Amikam,Y., Afik,S., Ofir,G., Sorek,R., Charpak-Amikam,Y., et al. (2015) BREX is a novel phage resistance

341 9. Cohen,D., Melamed,S., Millman,A., Shulman,G., Oppenheimer-Shaanan,Y., Kacen,A., 342 Doron,S., Amitai,G. and Sorek,R. (2019) Cyclic GMP-AMP signalling protects bacteria 
against viral infection. Nature, 574, 691-695.

344 10. Millman,A. (2020) Bacterial retrons function in anti-phage defense. Cell, 183, 1551-1561.e12.

345 11. Rousset,F., Dowding,J., Bernheim,A., Rocha,E.P.C. and Bikard,D. (2021) Prophage-encoded

346 hotspots of bacterial immune systems. bioRxiv, 10.1101/2021.01.21.427644.

347 12. Bernheim,A., Millman,A., Ofir,G., Meitav,G., Avraham,C., Shomar,H., Rosenberg,M.M., Tal,N., Melamed,S., Amitai,G., et al. (2021) Prokaryotic viperins produce diverse antiviral molecules. Nature, 589, 120-124.

13. Ofir,G., Melamed,S., Sberro,H., Mukamel,Z., Silverman,S., Yaakov,G., Doron,S. and Sorek,R. (2018) DISARM is a widespread bacterial defence system with broad anti-phage activities. Nat. Microbiol., 3, 90-98. Phage on tap-a quick and efficient protocol for the preparation of bacteriophage laboratory stocks. PeerJ, 4, e2261.

15. Clokie,M.R.J. and Kropinski,A.M. (2009) Bacteriophages. Methods and Protocols Volume 1:

16. Sambrook,J. and Russell,D. (2011) Purification of Nucleic Acids by Extraction with Phenol:Chloroform. Cold Spring Harb. Protoc., 2006, 4455.

17. McAlister,V., Zou,C., Winslow,R.H. and Christie,G.E. (2003) Purification and in vitro characterization of the Serratia marcescens NucC protein, a zinc-binding transcription factor homologous to P2 Ogr. J. Bacteriol., 185, 1808-1816.

18. Feng,Z., Fang,G., Korlach,J., Clark,T., Luong,K., Zhang,X., Wong,W. and Schadt,E. (2013) Dependence of Polymerase Kinetic. PLoS Comput. Biol., 9, 1-10. 
and Brouns,S.J.J. (2018) Targeting mechanisms of tailed bacteriophages. Nat. Rev. Microbiol., 16, 760-773.

20. Howard-Varona,C., Hargreaves,K.R., Abedon,S.T. and Sullivan,M.B. (2017) Lysogeny in nature: Mechanisms, impact and ecology of temperate phages. ISME J., 11, 1511-1520.

21. Venclovas, Ц̌ . (2019) Genome Replication of Bacterial and Archaeal Viruses. In Bamford,D.H., Zuckerman,M. (eds.), Encyclopedia of Virology (Fourth Edition). Academic Press, pp. 429-

22. Trun,N. and Trempy,J. (2009) Fundamental Bacterial Genetics Wiley. Wiley-Blackwell.

23. Gordeeva,J., Morozova,N., Sierro,N., Isaev,A., Sinkunas,T., Tsvetkova,K., Matlashov,M., Truncaite,L., Morgan,R.D., Ivanov,N. V., et al. (2019) BREX system of Escherichia coli

24. Pleška,M. and Guet,C.C. (2017) Effects of mutations in phage restriction sites during escape from restriction-modification. Biol. Lett., 13, 9-12.

25. Murphy,J., Mahony,J., Ainsworth,S., Nauta,A. and van Sinderen,D. (2013) Bacteriophage orphan DNA methyltransferases: Insights from their bacterial origin, function, and

26. Pleška,M., Qian,L., Okura,R., Bergmiller,T., Wakamoto,Y., Kussell,E. and Guet,C.C. (2016) occurrence. Appl. Environ. Microbiol., 79, 7547-7555. Structural basis for broad anti-phage immunity by DISARM. bioRxiv. 
bioRxiv preprint doi: https://doi.org/10.1101/2021.12.28.474362; this version posted December 28, 2021. The copyright holder for this preprint (which was not certified by peer review) is the author/funder, who has granted bioRxiv a license to display the preprint in perpetuity. It is made available under aCC-BY-NC-ND 4.0 International license.

391 29. Fomenkov,A., Sun,Z., Murray,I.A., Ruse,C., Mcclung,C., Yamaichi,Y., Raleigh,E.A. and

392 Roberts,R.J. (2020) Plasmid replication-associated single-strand-specific methyltransferases.

$393 \quad$ Nucleic Acids Res., 48, 12858-12873.

394

395 
bioRxiv preprint doi: https://doi.org/10 1101/2021.12.28.474362. this version posted December 28, 2021. The copyright holder for this preprint (which was not certified by peer review) is the author/funder, who has granted bioRxiv a license to display the preprint in perpetuity. It is made available under aCC-BY-NC-ND 4.0 International license.

\section{FIGURES}

A Serratia sp. SCBI
NZ_CP003424.1
$(535783: 550823)$
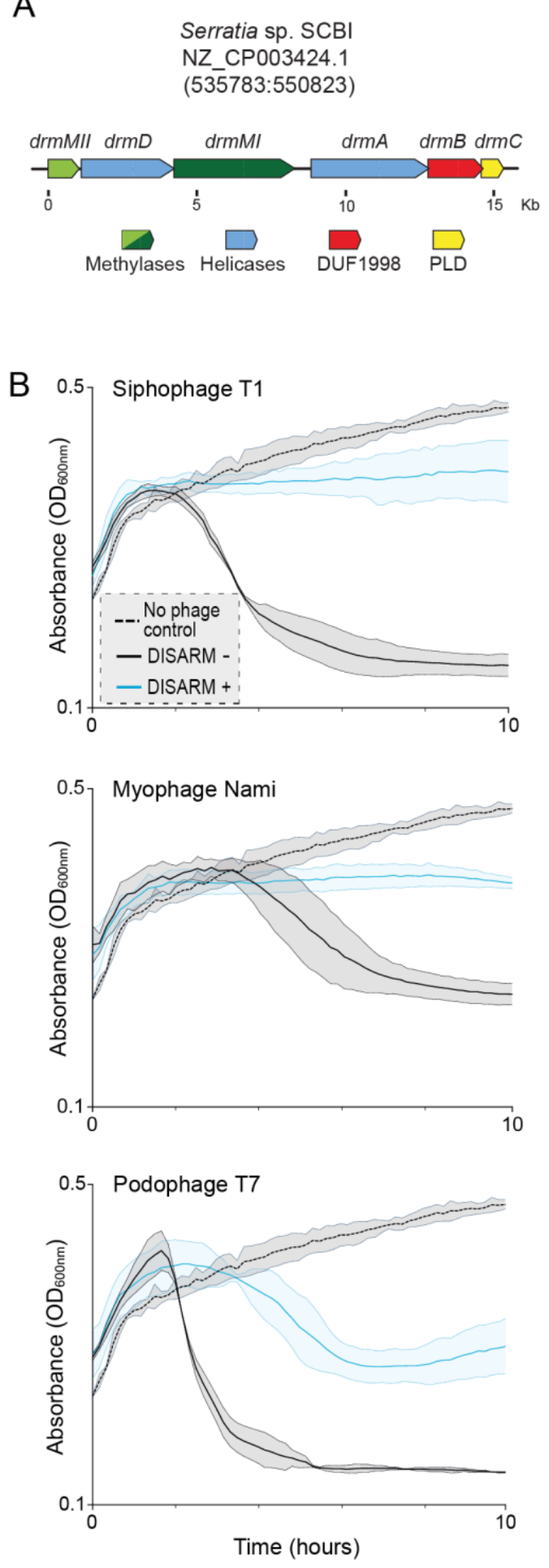
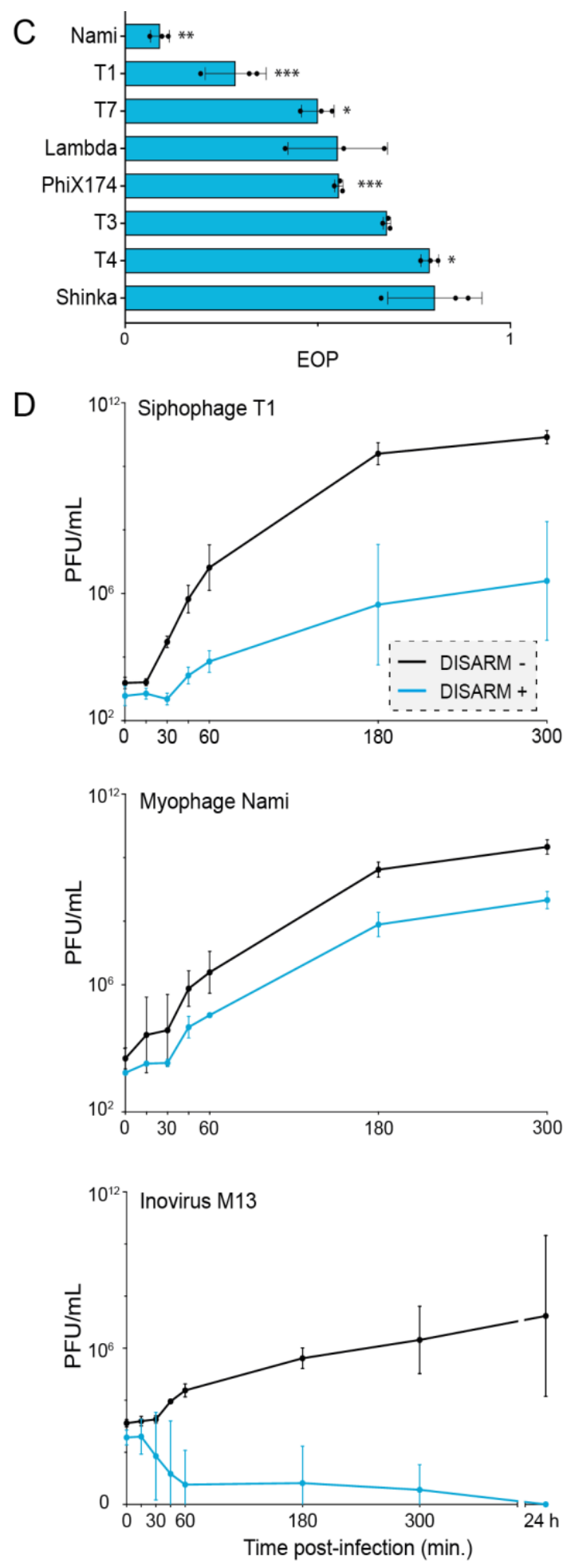
398 Figure 1. Protection provided by Class 1 DISARM against phage infection. (A) Gene cluster of

399 Class 1 DISARM from Serratia sp. SCBI. (B) Effect of siphophage T1, myophage Nami, and 400 podophage T7 on the growth curve of DISARM (+) or DISARM (-) strains. Uninfected DISARM 401 (-) and DISARM (+) strains have similar growth curves and only uninfected DISARM (-) is 402 displayed. Full results can be seen in Supplementary Figure S2. Initial MOI: 5x10-4 . Filled areas 403 inside dotted lines indicate standard deviation of three independent replicates. (C) Efficiency of 404 plating (EOP) of a set of DNA phages in a DISARM (+) strain normalized to the DISARM (-) 405 strain. (D) Effect of DISARM on the population of siphophage T1, myophage Nami, and inovirus 406 M13 over time. Bacterial cultures of DISARM (-) or DISARM (+) strains were infected with phage 407 at MOI of $2 \times 10^{-6}, 1 \times 10^{-5}$ and $2 \times 10^{-6}$ for phages T1, Nami and M13 respectively, and the titer was 408 determined at selected time points. Curves depict the average and standard deviation of three 409 independent experiments. Statistical significance was determined by two-tailed, unpaired t Test 410 and is represented as $* * *$, or $* * *$ for $\mathrm{p}<0.05,0.01$ or 0.001 , respectively. 

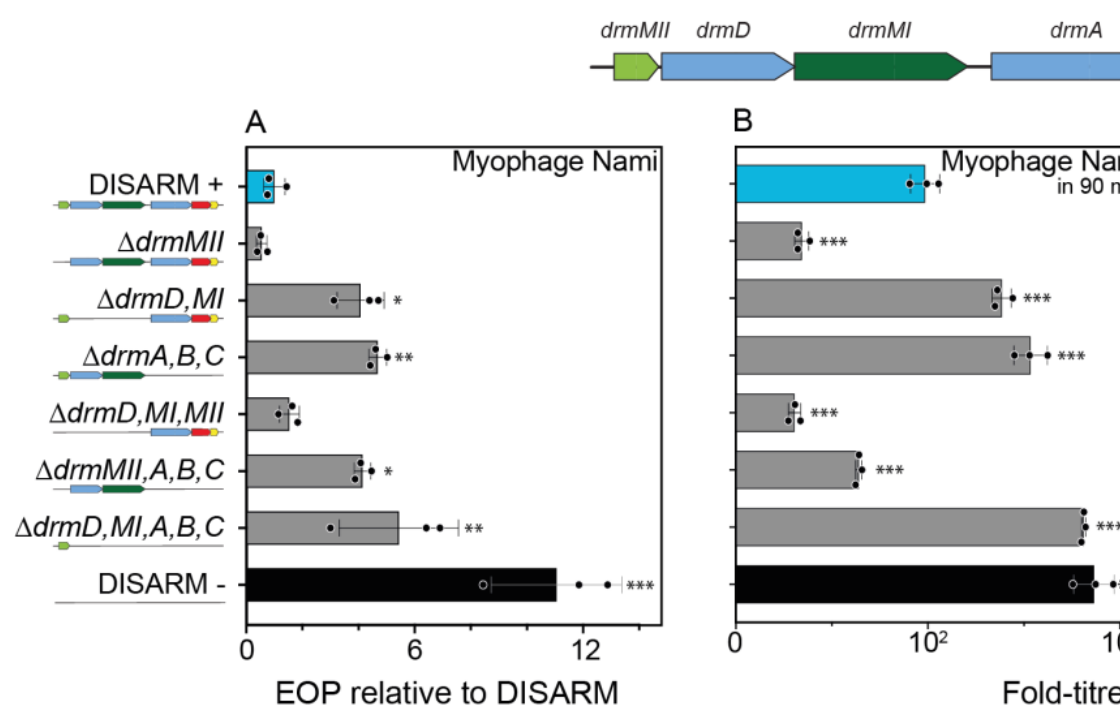

$\operatorname{drmB} \operatorname{drmC}$
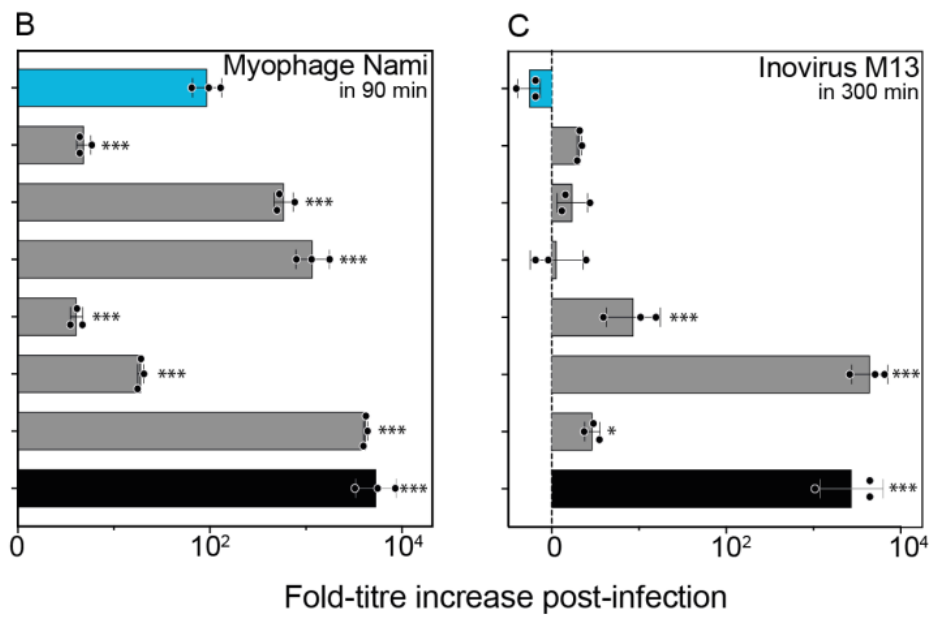

412 Figure 2. Effect of Class 1 DISARM components on protection against phage infection. (A)

413 Efficiency of plating (EOP) of myophage Nami on strains containing the modified DISARM

414 system (DISARM (-) strain), normalized to the DISARM strain. Titer-fold increase of (B)

415 myophage Nami, and $(\mathbf{C})$ inovirus M13 upon propagation in cultures of strains containing the

416 complete or modified DISARM system. Graphics represent the time point at which maximum

417 effect on phage replication was achieved. For $(\mathbf{C})$, negative values indicate phage titers below the

418 initial phage titer. Bars depict the average and standard deviation of three independent replicates.

419 Statistical significance was determined by one-way ANOVA+ with Tukey post-hoc test and is

represented as $*, * *$, or $* * *$ for $\mathrm{p}<0.05,0.01$ or 0.001 , respectively. 

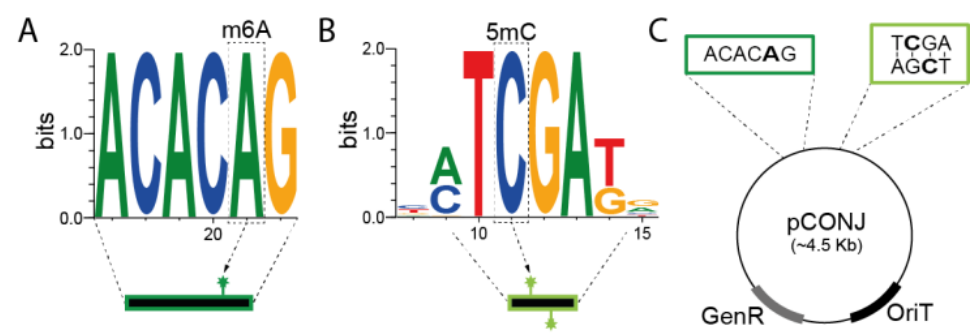

D
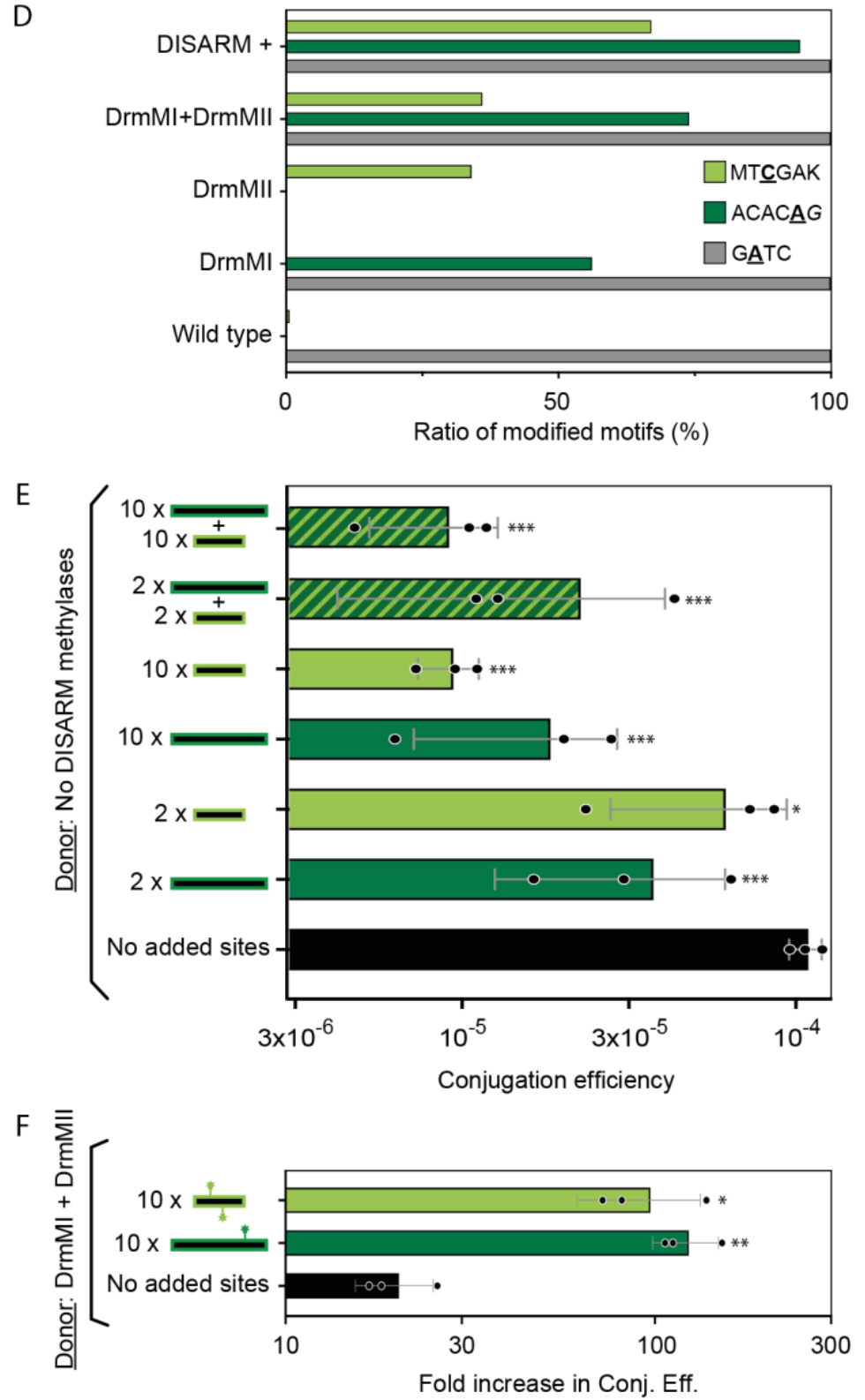

422 Figure 3. Effect of DNA methylation in Class 1 DISARM. Weblogos of methylation motifs of

423 DISARM methylases (A) DrmMI and (B) DrmMII. (C) Schematic representation of pCONJ

424 plasmid with motifs as defined in (A) and (B). (D) Relative number of modified sites detected in 
425 the genome of E. coli expressing the Class 1 DISARM system of Serratia sp. SCBI. Three distinct

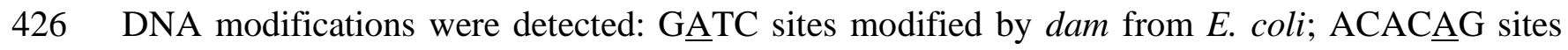

427 modified by $d r m M I$; and MTCGAK sites modified by drmMII. (E) Conjugation efficiency of

428 plasmid pCONJ with variable numbers of unmethylated motifs ACAC $\underline{A G}$ and/or MTCGGAK into

429 the recipient DISARM (+) strain (see Supplementary Figure S5B for results in recipient control

430 strain). Control is pCONJ with no motifs in its sequence. (F) Conjugation efficiency of plasmid

431 pCONJ with 10 methylated motifs ACACÁG or MTCGGAK into the recipient DISARM (+) strain,

432 normalized by conjugation efficiency of pCONJ with unmethylated motifs. 


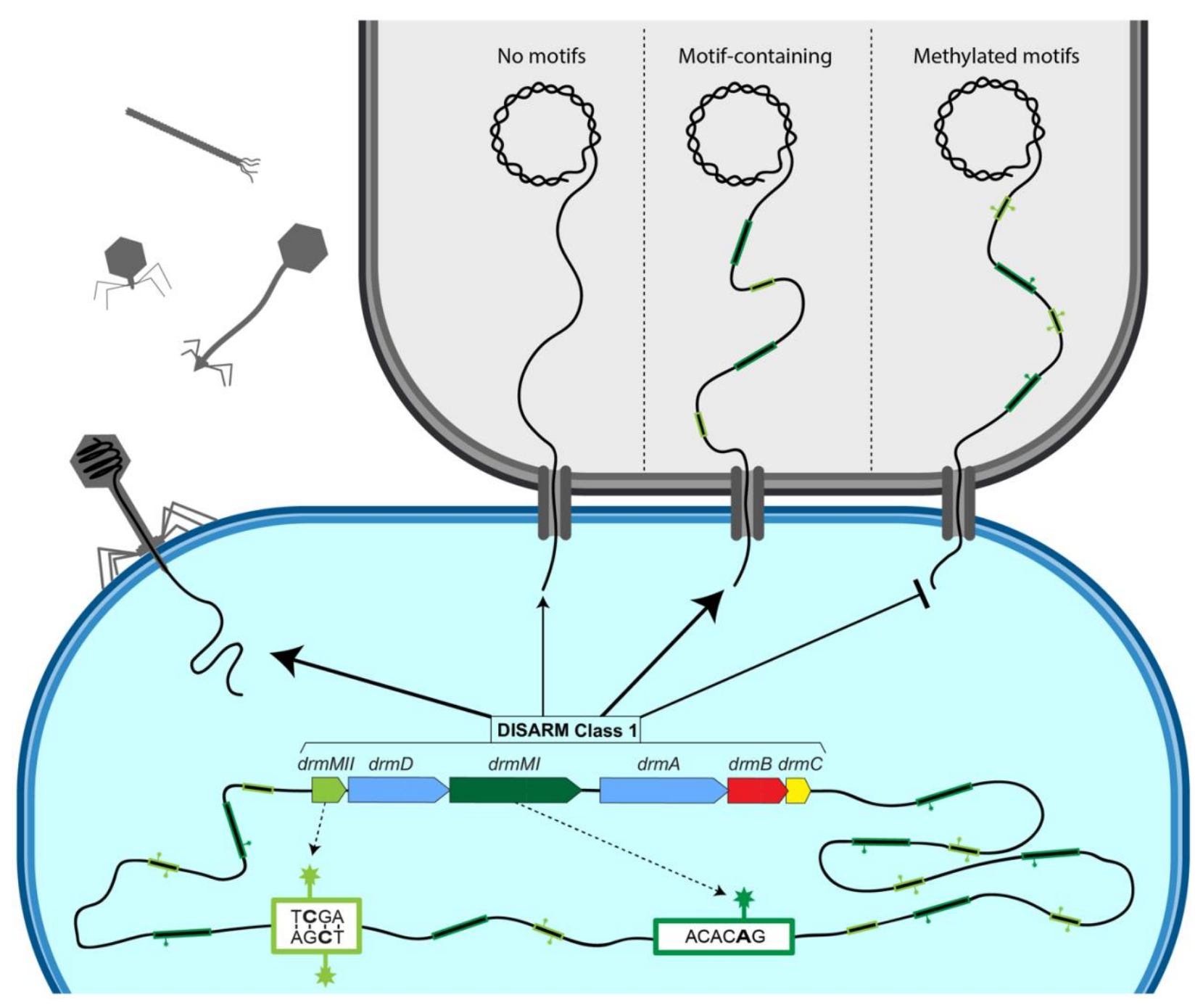

434 Figure 4. Mechanism of action of Class 1 DISARM of Serratia sp. SCBI. The methylases drmMI

435 and drmMII methylate the host DNA at motifs ACAC $\underline{A G}$ and MTCGAK, respectively. The

436 DISARM system provides protection from incoming unmethylated plasmid DNA and is less active

437 on incoming DNA with methylated DISARM motifs. The efficiency of the protection increases

438 with the number of unmethylated motifs present in the conjugated plasmid DNA. 